\title{
Folate deficiency in rat pups during weaning causes learning and memory deficits
}

\author{
Maria I. Berrocal-Zaragoza ${ }^{1,2,3}$, Jeffrey M. Sequeira ${ }^{1}$, Michelle M. Murphy ${ }^{2,3}$, Joan D. Fernandez- \\ Ballart $^{2,3}$, Samah G. Abdel Baki ${ }^{4}$, Peter J. Bergold ${ }^{4}$ and Edward V. Quadros ${ }^{1 *}$ \\ ${ }^{1}$ Department of Medicine, State University New York (SUNY)-Downstate Medical Center, Box 20, 450 Clarkson Avenue, \\ Brooklyn, NY 11203, USA \\ ${ }^{2}$ Preventive Medicine and Public Health Unit and IISPV Faculty of Medicine and Health Sciences, \\ Universitat Rovira $i$ Virgili, Reus, Tarragona, Spain \\ ${ }^{3}$ CIBER Fisiopatología de la Obesidad y Nutrición (CBO6/03), Instituto Carlos III, Spain \\ ${ }^{4}$ Department of Physiology and Pharmacology, State University New York (SUNY)-Downstate Medical Center, \\ Brooklyn, NY, USA \\ (Submitted 25 December 2013 - Final revision received 14 May 2014-Accepted 26 June 2014 - First published online 3 September 2014)
}

\begin{abstract}
Folate is essential for fetal development, and its deficiency during gestation causes behavioural deficits in the offspring. The present study investigated its influence during weaning on brain function in the pups of rats that were put on a folate-deficient (FD) diet on postnatal day (PND) 1. Systemic folate deficiency in pups on the FD diet $(n 15)$ was evident from the dramatically lower hepatic folate concentrations (median $23 \cdot 7$, range $8 \cdot 1-48.4 \mathrm{ng} / \mathrm{mg}$ protein) and higher homocysteine concentrations (median $27 \cdot 7$, range $14 \cdot 7-45 \cdot 5 \mathrm{pmol} / \mathrm{mg} \mathrm{protein}$ ), respectively, compared with those of pups on the normal diet (ND; $n$ 9) (median 114.5, range 64.5-158.5 ng/mg protein and median 15.5 , range $11.6-18.9 \mathrm{pmol} / \mathrm{mg}$ protein) on PND 23. Brain folate concentrations although low were similar in pups on the FD diet (median 10.5, range $5 \cdot 5-24 \cdot 5 \mathrm{ng} / \mathrm{mg}$ protein) and ND (median $11 \cdot 1$, range $7 \cdot 1-24 \cdot 2 \mathrm{ng} / \mathrm{mg}$ protein). There was a high accumulation of homocysteine in the brain of FD pups, mostly in the hippocampus (median 58.1, range $40 \cdot 8-99 \cdot 7 \mathrm{pmol} / \mathrm{mg}$ protein) and cerebellum (median $69 \cdot 1$, range $50 \cdot 8-126 \cdot 6 \mathrm{pmol} / \mathrm{mg}$ protein), indicating metabolic folate deficiency despite normal brain folate concentrations. Developmental deficits or autistic traits were more frequent in the FD group than in the ND group and repetitive self-grooming occurred, on average, three times (range 1-8) $v$. once (range 0-3) during $5 \mathrm{~min}$, respectively. Long-term memory or spatial learning and set-shifting deficits affected 12 to $62 \%$ of rats in the FD group compared with none in the ND group. Post-weaning folic acid supplementation did not correct these deficits. These observations indicate that folate deficiency during weaning affects postnatal development even when gestational folate supply is normal.
\end{abstract}

\section{Key words: Folate receptors: Weaning period: Behaviours: Brain function: Set shifting}

Folate functions in the transfer of carbon units in metabolic pathways essential for the interconversion of amino acids, synthesis of nucleic acids, transfer of methyl groups, and regulation of gene expression and neurotransmitter synthesis ${ }^{(1)}$. The essential role of folate during embryogenesis and fetal development is well established from human studies ${ }^{(2,3)}$ and from the mouse folate receptor- $\alpha$ gene knockout model ${ }^{(4)}$. However, its role in the development of the central nervous system in late pregnancy and following birth is yet to be defined. The brain of a newborn rat continues to develop and establish functional plasticity following birth. Early postnatal development of the rat brain corresponds to late gestation in humans ${ }^{(5)}$. This period is characterised by rapid development and maturation of motor coordination and sensory systems that underlie refinements of social behaviour and communication. Stead et $a l^{(6)}$ showed a significant increase in the expression and reorganisation of groups of genes in rat brain related to specific functions such as myelination and synaptic vesicle transport during early postnatal life. The expression of specific genes was found to exhibit a spatiotemporal pattern in the hypothalamus, hippocampus and frontal cortex throughout different stages of development. Maternal folate deficiency in late pregnancy was found to decrease the number of progenitor cells in the mitotic phase and increase the number of apoptotic cells and the concentration of calretinin, a Ca-binding protein present in neurons

Abbreviations: FD, folate deficient; ND, normal diet; PND, postnatal day.

*Corresponding author: E. V. Quadros, fax +1 718270 1578, email edward.quadros@downstate.edu 
involved in attention and memory process in mouse fetal forebrain $^{(7)}$. Because of the central role of folate in DNA synthesis and maintenance of methylation status, folate deficiency could have lasting effects that could be transmitted across generations. These effects are most felt during pregnancy and could contribute to overall health of the offspring during the course of its life ${ }^{(8)}$.

Very little is known about the effects of folate on brain development and function after birth ${ }^{(9)}$. Folate deficiency in rats has been shown to affect neural cell proliferation, vesicular transport and synaptic plasticity with long-term effects on learning and memory ${ }^{(10)}$. These pathologies may not present as gross morphological abnormalities, but may have a profound effect on proper development and function of the brain. Many of the developmental disorders in humans appear to manifest during the first and second years of life. Although numerous underlying causes may contribute to the pathology, inadequate supply of folate, which is critical for brain development during fetal life, may play an important role in postnatal structural and functional refinement of the brain. In this regard, maternal folate status during the breastfeeding period is an important determinant of folate available to the infant. The aims of the present study were to determine the effect of folate deficiency during the weaning period on folate status in rat pups and to evaluate what effect this transient folate deficiency would have on behaviour, learning and cognition in adult life.

\section{Experimental methods}

\section{Animals and study design}

A flowchart of the study design is shown in Fig. 1. A total of eight pregnant dams (Long Evans hooded rats purchased from Charles River Laboratories International, Inc., Wilmington, MA, USA) were fed a normal diet (ND) containing $2 \mathrm{mg}$ folic $\mathrm{acid} / \mathrm{kg}$ chow (Dyets, Inc.) as recommended by the American Institute of Nutrition ${ }^{(11)}$. Immediately after giving birth, the dams and their pups were separated into two groups:

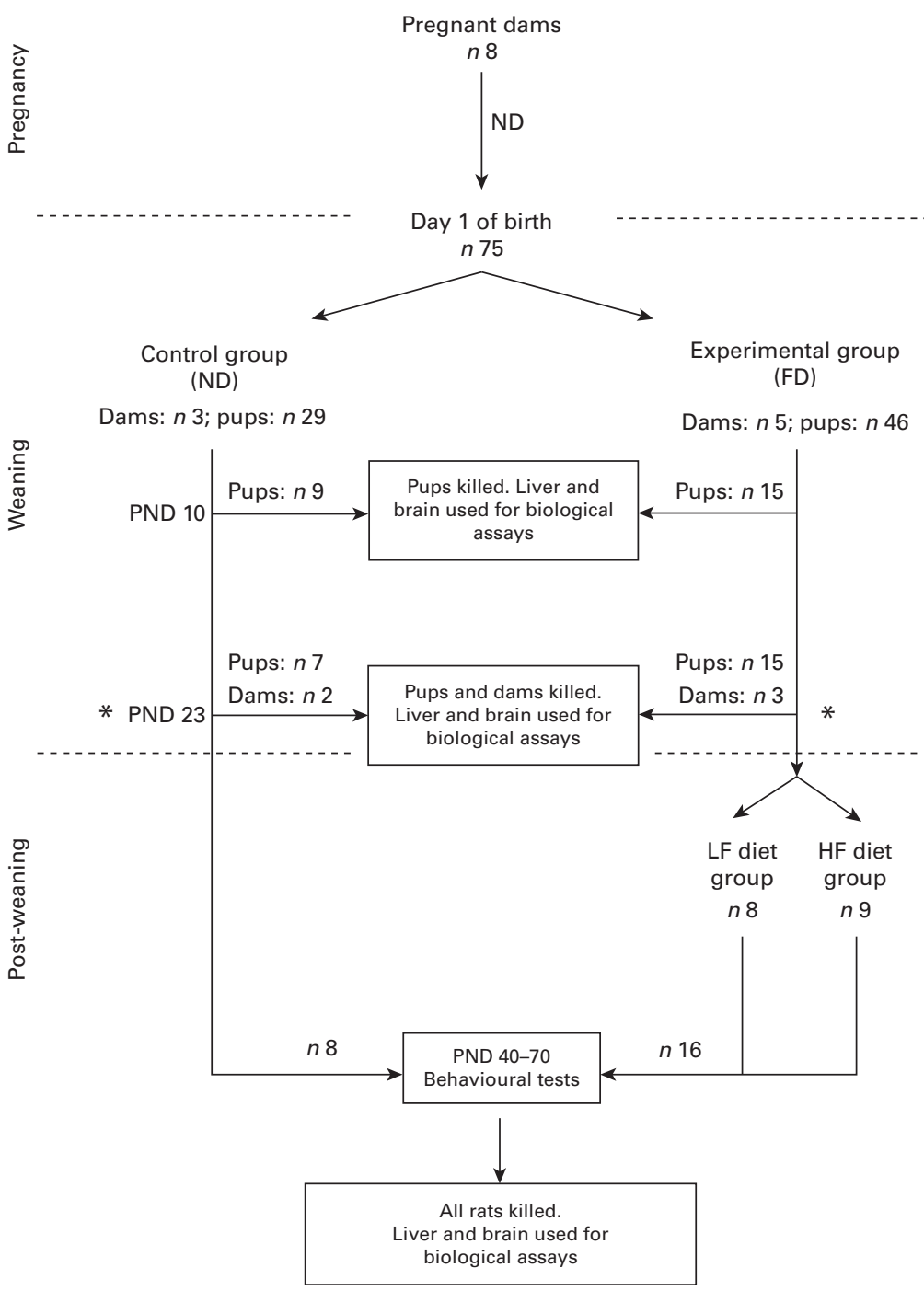

Fig. 1. Flowchart of the study design. On postnatal day (PND) 23, dams were separated from their pups. ND, normal diet (containing $2 \mathrm{mg}$ folic acid/kg chow); $\mathrm{FD}$, folate-deficient diet (with $1 \%$ succinyl sulfathiazole); LF, low folate (FD: $6 \mathrm{~d} /$ week and ND: $1 \mathrm{~d} /$ week); HF, high folate (ND plus $1 \mathrm{mg} / \mathrm{ml}$ folic acid in water). 
one group of three dams and their pups (totally twenty-nine pups) were continued on the ND (control group) and another five dams and their pups (totally forty-six pups) were put on a folate-deficient (FD) diet with $1 \%$ succinyl sulfathiazole (Dyets, Inc.) to prevent folate synthesis by the bacterial flora of the gastrointestinal tract for the entire weaning period (the first $23 \mathrm{~d}$ after birth). Some of the pups from the control group ( $n$ 9) and from the experimental group ( $n$ 15) were killed on postnatal day (PND) 0. Additional pups and dams of both groups (control: two dams and seven pups; experimental: three dams and fifteen pups) were killed on PND 23 (end of the weaning period). Livers and brains were collected for the determination of folate and homocysteine concentrations. Post-weaning, after the pups were separated from the dams, the pups from the control group ( $n$ 13) continued be fed the ND, while the pups from the experimental group ( $n$ 16) were placed on two different new diets: one half of the pups ( $n$ 8) were maintained on a low-folate diet (FD: $6 \mathrm{~d} /$ week and ND: $1 \mathrm{~d} /$ week) and the other half $(n 8)$ were maintained on a high-folate diet (ND plus $1 \mathrm{mg} / \mathrm{ml}$ folic acid in water). The pups were raised on these diets before behavioural tests between PND 40 and 70 for parameters such as anxiety (open field) and place avoidance tasks for spatial memory formation. Finally, they were killed and their livers and brains were collected for folate determination. All rats had free access to food and water. Weight gain in pups during and after weaning was monitored. They were maintained at a room temperature of $22^{\circ} \mathrm{C}$ under a $12 \mathrm{~h}$ light $-12 \mathrm{~h}$ dark cycle. The experimental protocol was approved by the Institutional Animal Care and Use Committee of SUNY-Downstate Medical Center. All procedures involving animals were conducted in conformity with state and federal laws and the standards outlined in the US National Institutes of Health Guide for the care and use of laboratory animals.

\section{Folate and homocysteine assays}

Brains and livers were homogenised in three volumes/weight of $0.5 \%$ ascorbic acid in PBS. For folate extraction, an aliquot of the homogenised tissue was diluted 16-fold with $2 \%$ ascorbic acid ( $\mathrm{pH}$ 5.5) and placed in a boiling water-bath for $15 \mathrm{~min}$. The precipitated protein was removed by centrifugation and the supernatant was used for the folate assay. A sequential binding radioassay with $N^{5}$-methyltetrahydrofolate as the standard, ${ }^{3} \mathrm{H}$ pteroylglutamic acid (Moravek) as the radiolabelled tracer and purified human milk folate receptor- $\alpha$ as the binding protein was used for the determination of folate concentrations in tissues ${ }^{(12)}$. Total protein concentrations were determined using the Bio-Rad Protein Assay (Bio-Rad Laboratories, Inc.), and folate concentrations are expressed in ng folate/mg protein. Brains were dissected to isolate different regions for this assay. Homocysteine concentrations in the liver and brain tissues on PND 23 were determined using the Axis Homocysteine EIA Kit (Axis Shield). Tissue samples were homogenised in three volumes of PBS containing $5 \mathrm{~mm}$-dithiothreitol, membranes were pelleted by centrifugation at $15000 \mathrm{~g}$ for $5 \mathrm{~min}$ and $50 \mu \mathrm{l}$ of the supernatant were used in the assay. Homocysteine concentrations are expressed in $\mathrm{pmol} / \mathrm{mg}$ protein. The weighted averages of folate and homocysteine concentrations in the whole brain were calculated by taking into account the relative volume of each region of the brain ${ }^{(13,14)}$.

\section{Behavioural tests}

A total of twenty-four rats were tested (sixteen rats from the experimental group (eight from the low-folate diet group and eight from the high-folate diet group after weaning) and eight rats from the control group) on PND 40-45 for the open field test and on PND 57-70 for the place avoidance tests.

\section{Open field test}

The open field test is used to evaluate anxiety, locomotor activity and exploratory behaviours ${ }^{(15)}$. Each rat was placed in the centre of an open field $(60 \mathrm{~cm} \times 60 \mathrm{~cm}$ with $40 \mathrm{~cm}$ high walls) that was divided into a grid of $5 \times 5 \mathrm{~cm}$ squares. Movement and behaviour of the rat in this arena during a 5 min session were recorded using an overhead camera. After $5 \mathrm{~min}$, the rat was returned to the cage and the open field arena was cleaned with $30 \%$ ethanol to prevent olfactory cues from affecting the behaviour of subsequently tested rats. An observer blinded to the experimental conditions viewed the video recording of each rat for distance travelled (cm) by counting the number of squares crossed during each session and time (s) spent in the peripheral, central and corner areas of the field, the number of rearings (times/ $5 \mathrm{~min}$ ) and self-groomings (times $/ 5 \mathrm{~min}$ ), and the total number of faecal pellets.

\section{Place avoidance tests}

These validated behavioural tests are used to evaluate learning, memory and cognition as described previously ${ }^{(16,17)}$. The tests consist of different phases that are performed over a period of three consecutive days: passive place avoidance $(4 \times 10 \mathrm{~min}$ trials $)$ - rats learnt a stationary shock zone location in a stationary arena using olfactory and visual cues; active place avoidance $(6 \times 10$ min trials $)$ - rats learnt to avoid a stationary shock zone in a rotating arena by segregating stationary visual cues from the rotating local olfactory cues $^{(18,19)}$; conflict place avoidance $(6 \times 10$ min trials $)$. Only rats that successfully learnt active place avoidance and could remember the task the next day were allowed to undergo these trials and their ability to avoid a new location in a rotating arena was tested.

Parameters acquired and analysed during each trial included the following: total distance travelled (m); number of entrances into the shock zone; number of shocks received; distance $(\mathrm{m})$ of avoidance; latency to first entrance into the shock sector. Success at completing each task was monitored by noting the ability of rats to demonstrate clear knowledge of the shock zone by significantly increasing the time to first entry and decreasing the number of entrances into the shock zone with successive trials. 


\section{Statistical analysis}

The non-parametric one-tailed Mann-Whitney $U$ test was used to compare folate concentrations on PND 10 and PND 23 between the ND and FD groups, folate and homocysteine concentrations on PND 23 between the ND and FD groups, and folate concentrations at the end of the behavioural tests among the ND, low-folate diet and high-folate diet groups. The Mann-Whitney $U$ test was used to compare the weight of pups between the ND and FD groups on PND 10 and 23.

Non-parametric tests were used because the sample size was small. Quantitative variables were compared with the Mann-Whitney $U$ test to report the median, the $Z$ value and the $P$ value, when the difference between the groups was significant or almost significant. The same test was used to compare behavioural variables of the open field test. A cross-sectional analysis and a longitudinal analysis of variables of the place avoidance tasks were conducted. In the crosssectional analysis, the results of the parameters in each trial of the passive, active and conflict place avoidance tasks were compared between the control and experimental groups, the control and low-folate diet groups, the control and high-folate diet groups, and finally the low-folate diet and high-folate diet groups. The results obtained in each trial of the passive, active and conflict place avoidance tasks were longitudinally analysed in each of these groups using the Wilcoxon signed-rank test. The results obtained in the first trial of each phase of the place avoidance task were compared with those of the rest of the trials (trial $1 v$. trial 2, trial 1 $v$. trial 3, etc.) and those of each trial with those of the following one (trial $1 v$. trial 2 , trial $2 v$. trial 3 , etc.) for the three place avoidance phases. Bonferroni correction was applied to the $P$ values for multiple comparisons. The $\chi^{2}$ test was used to compare rats that succeeded at completing each phase of the place avoidance task between the ND and FD groups. The significance level for all tests was set at $P<0.05$. The Statistical Package for the Social Sciences (version 19.0; SPSS, Inc.) was used for the statistical analysis.

\section{Results}

Hepatic folate concentrations ranged from 62 to $253 \mathrm{ng} / \mathrm{mg}$ protein, and the median concentration was lower in ND pups than in FD pups on PND 10. This changed by PND 23 with hepatic folate concentrations showing a significant drop of more than $75 \%$ in FD pups. However, brain folate concentrations were similar in both the ND and FD groups even though folate concentrations had dropped by about $70 \%$ between PND 10 and 23 in both groups (Table 1). The distribution of folate within different regions of the brain was similar in both the ND and FD groups on PND 10. This profile extended to PND 23 with decreases in all regions being consistent with the decrease in the whole-brain total folate concentrations (Table 1). Pups reared by dams on the FD diet appeared to grow normally and had similar weights $(\mathrm{g})$ on PND 10 compared with pups on the ND (median, range, $n: 16 \cdot 9,12 \cdot 7-20 \cdot 9,15 v \cdot 17 \cdot 8,15 \cdot 6-20 \cdot 2$, 9), but FD pups had significantly lower weight on PND 23 than ND pups 


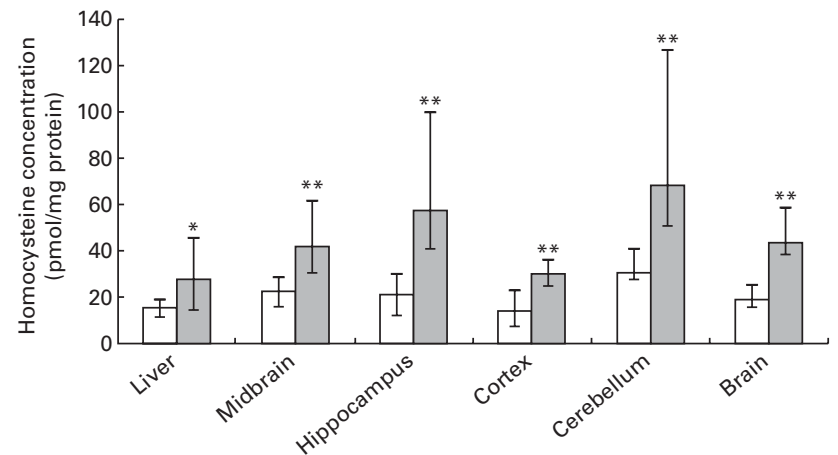

Fig. 2. Homocysteine concentrations in the liver, brain and different regions of the brain in rat pups on postnatal day 23. Values are medians and ranges represented by vertical bars. $\square$, Rats on a normal diet $(n 5)$ containing $2 \mathrm{mg}$ folic acid/kg chow; $\square$, rats on a folate-deficient diet $(n 7)$ with $1 \%$ succinyl sulfathiazole throughout the weaning period (until postnatal day 23). Median was significantly different from that of the normal diet group: ${ }^{*} P<0.05$, ${ }^{\star *} P<0.01$.

$(48 \cdot 9, \quad 41 \cdot 9-57 \cdot 8, \quad 15 \quad v . \quad 57 \cdot 4, \quad 49 \cdot 5-72 \cdot 1, \quad 7 ; \quad Z=-2 \cdot 820$, $P=0 \cdot 003)$. This difference was corrected once the pups were switched to a low-folate diet or a high-folate diet after PND 23 and the weights were similar in both groups between PND 40 and 70. Hepatic folate concentrations in the three dams on the FD diet (27, 45 and $25 \mathrm{ng} / \mathrm{mg}$ protein) were 4-fold lower than those in the two control dams on the ND (172 and $132 \mathrm{ng} / \mathrm{mg}$ protein) on PND 25. Brain folate concentrations were similar in both FD (17, 23 and $48 \mathrm{ng} / \mathrm{mg}$ protein) and ND (30 and $25 \mathrm{ng} / \mathrm{mg}$ protein) dams. On PND 70, as expected, hepatic folate concentrations ( $\mathrm{ng} / \mathrm{mg}$ protein) in low-folate diet rats were significantly lower than those in high-folate diet rats (median, range, $n: 31 \cdot 2,14 \cdot 1-59 \cdot 2,8, v$. $215 \cdot 0,133 \cdot 8-289 \cdot 1,8 ; Z=-3 \cdot 36, P<0 \cdot 001)$. Brain folate concentrations also differed significantly between the low-folate diet and high-folate diet groups (median, range, $n: 21 \cdot 7$, $10 \cdot 0-70 \cdot 3,8$ v. 117.4, 85.0-178.9, 8; $Z=-2 \cdot 700, P=0 \cdot 003$ ). Tissue homocysteine concentrations on PND 23 indicated a significant increase in hepatic homocysteine concentrations in FD pups (Fig. 2), consistent with the $>75 \%$ decrease in hepatic folate concentrations observed. Homocysteine concentrations also increased in all regions of the brain in FD rats, despite a brain folate concentration that was similar to that in control ND rats. Within the brain, there was variation in the distribution of homocysteine, with the cortex having the lowest concentrations and the cerebellum having the highest concentrations in ND rats. In FD rats, the relative distribution of homocysteine within different regions of the brain was similar to that in ND rats, but the median brain homocysteine concentration in FD rats was double that observed in ND rats (Fig. 2).

There was a significant increase in the number of selfgroomings in the experimental group, weaned on the FD diet and then maintained on the low-folate or high-folate diet, than in the ND control group (Fig. 3). In the open field test, rats on the FD diet during weaning spent significantly more time in the peripheral area of the arena compared with those on the ND (time in s; median, range, $n: 141,59-174,9 v \cdot 127,73-194,13 ; Z=-1 \cdot 813, P=0.035)$ and they travelled a longer distance in the peripheral area compared with those on the ND (distance in $\mathrm{cm}$; median, range, $n: 176,624-2148,9$ v. 984, 516-1872, 13; $Z=-1.587, P=0.056)$.

Rats on the FD diet during weaning and then maintained on the low-folate or high-folate diet exhibited no significant differences in the place avoidance tasks. For this reason, the two groups were combined (as one FD experimental group) and compared with the ND control group. In the last trial of the passive place avoidance task, the experimental group entered the shock zone more times than the control group, but this was not statistically significant (Fig. 4). In this trial, the frequency at which rats were shocked was greater for the experimental group, but was not significant when Bonferroni correction was applied to the $P$ value. Rats that completed the place avoidance tasks in the experimental group performed the tasks successfully within the parameters and were able to learn each of the tasks within the number of trials used even though some of the parameters monitored were not similar to those for control rats. Overall, the experimental group exhibited an increase in the time to first entry in both the active and conflict avoidance tasks and the number of shocks received in the conflict avoidance task compared with the control groups.

Although all the rats in the control group were able to complete the place avoidance tasks, a number of rats in the experimental group failed one or more of the avoidance tasks and therefore did not advance to the next task. Failure in these tasks is defined as the inability to progress to the next task when the number of entrances into the shock zone does not decrease or time to entry into the shock zone does not increase over subsequent trials. Within the experimental group, the learning deficits varied with some being severely affected and unable to learn the simple passive avoidance task and others being mildly affected and failing in the active or conflict avoidance task. This was evident from the inability of rats to avoid the shock zone in subsequent trials

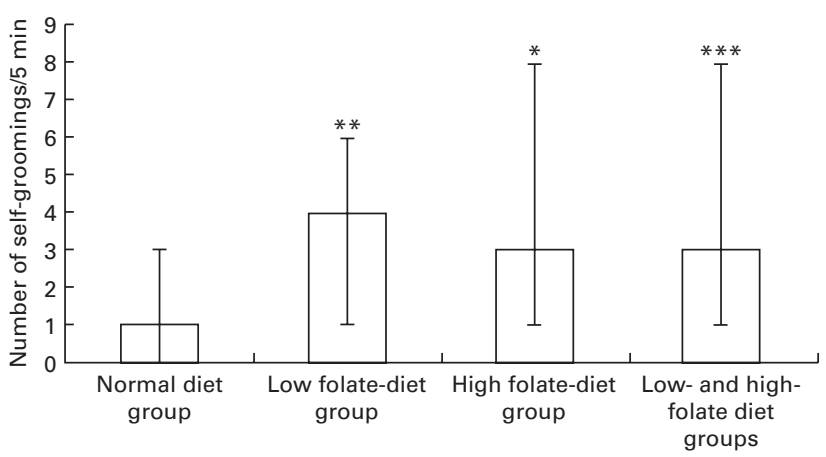

Fig. 3. Number of self-groomings in the open field test in rats on the lowfolate and high-folate diets. Values are medians and ranges represented by vertical bars. Normal diet group ( $n$ 13), rats on a normal diet throughout the weaning and post-weaning periods; low-folate diet group ( $n 4)$, rats on a folate-deficient diet until postnatal day 23 and then switched to a low-folate diet; high-folate diet group ( $n 5$ ), rats on a folate-deficient diet until postnata day 23 and then switched to a high-folate diet. Median was significantly different from that of the normal diet group: ${ }^{*} P<0.05,{ }^{* *} P<0.01$ ${ }^{\star * *} P<0.001$ 

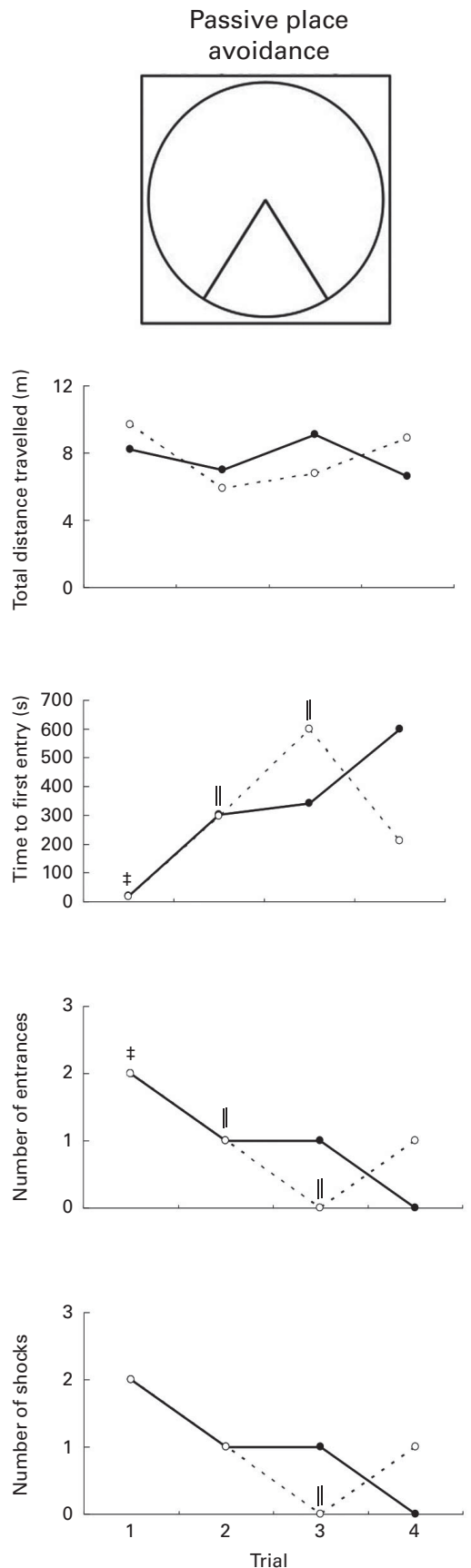
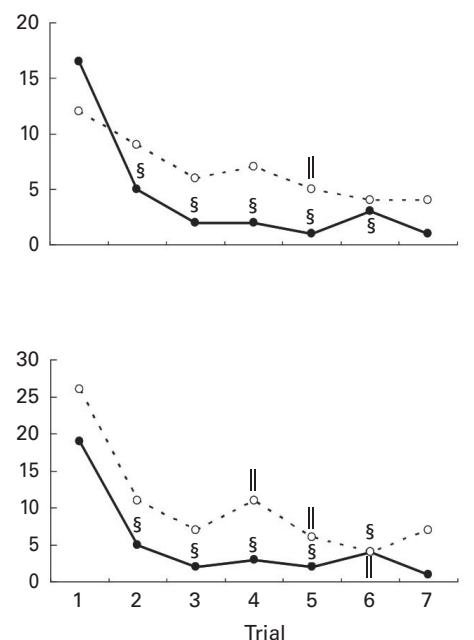

Conflict place

avoidance
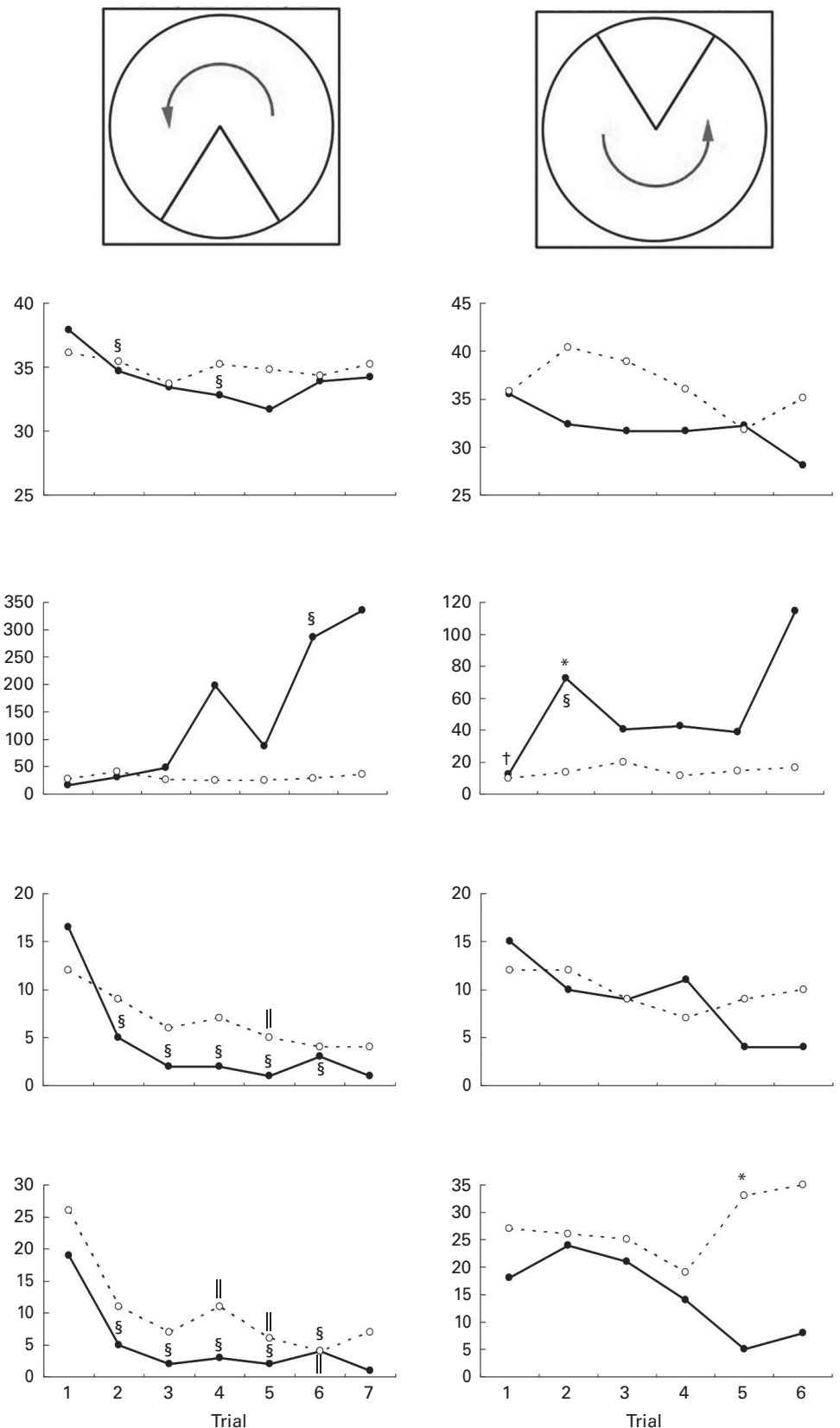

Fig. 4. Place avoidance parameters for different trials and tasks for rats fed a normal diet throughout the weaning and post-weaning periods (- $\bullet-;$ normal diet group; $n 8$ ) and rats on a folate-deficient diet until postnatal day 23 and then switched to a low-folate diet or a high-folate diet (-O-; folate-deficient groups; $n$ 16). Values are medians for the rats that successfully completed all the three tasks. Rats that failed one or more tasks are not included in this analysis. * Median was significantly different from that of the normal diet group $(P<0.05)$. Inter-trial parameters within each group of rats were compared. $\S$ Median of the normal diet group for the first trial of each phase of the place avoidance task was significantly different from those for the rest of the trials $(P<0.05)$. $\|$ Median of the folatedeficient groups for the first trial of each phase of the place avoidance task was significantly different from those for the rest of the trials $(P<0.05)$. $†$ Median of the normal diet group was significantly different from that for the following trial $(P<0.05)$. $¥$ Median of the folate-deficient groups was significantly different from that for the following trial $(P<0.05)$. Bonferroni correction was applied to the $P$ values for multiple comparisons.

as illustrated in trial 4 of the place avoidance tasks (Fig. 5). When the two groups were compared for all the behavioural tasks, there was considerable variation in the severity of affliction and, overall, $12 \%$ of rats in the experimental group failed the passive place avoidance task, 31\% failed the active place avoidance task and $62 \%$ failed the conflict place avoidance task (Fig. 6).

\section{Discussion}

The present study reports metabolic and developmental abnormalities in rat pups that did not receive adequate folate amounts from their mothers during weaning. These included homocysteine accumulation in the brain and developmental disorders such as autistic traits and reduced 


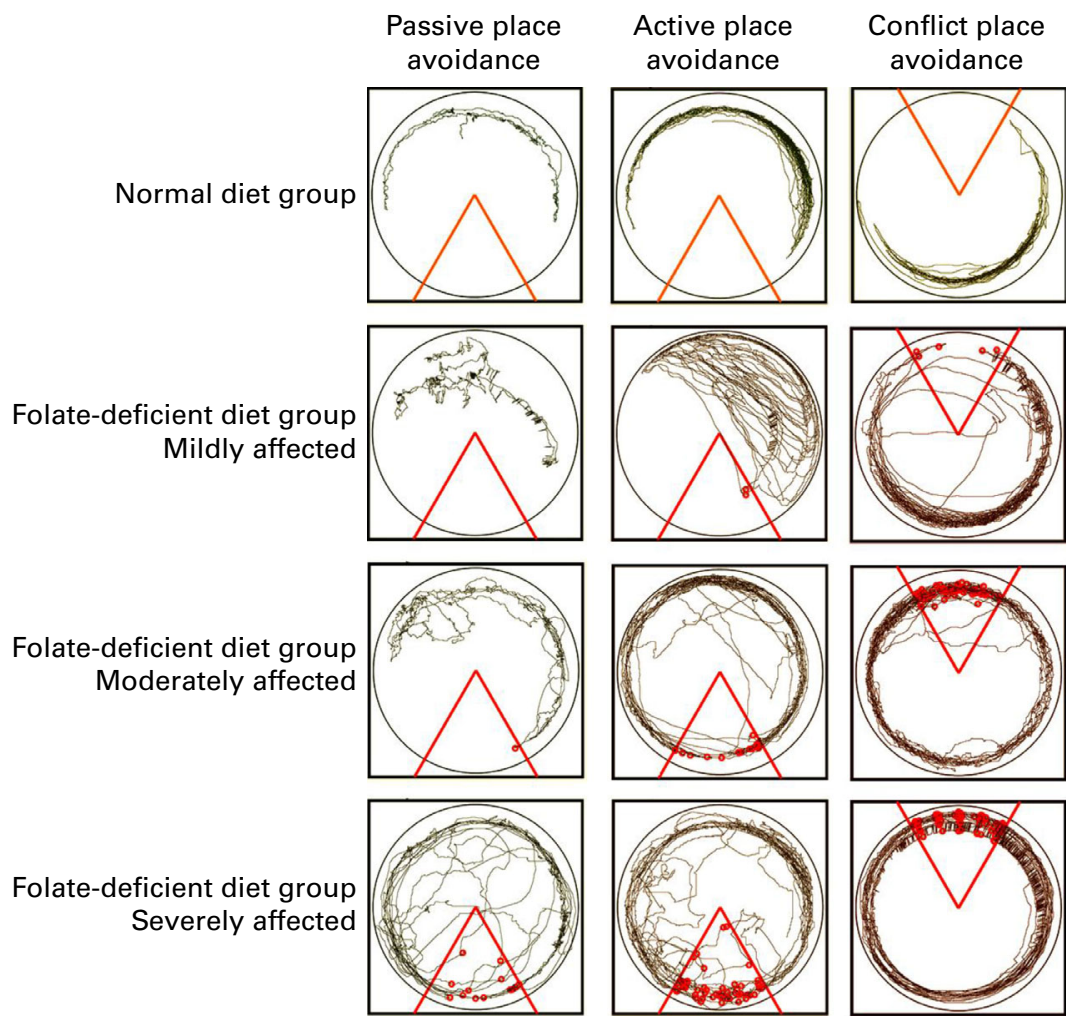

Fig. 5. Tracking of movements in the place avoidance tasks in the fourth trial for each of the three place avoidance tasks. Normal diet group: $n$; folate-deficient group: $n$ 16. The shock zone is indicated with a triangle within the circle and the dots within the triangle indicate shocks received. The tracing lines indicate movement of the rat within the circular platform. A colour version of this figure can be found online at http://www.journals.cambridge.org/bjn

long-term memory, compared with pups of dams on a normal folate diet. Post-weaning folic acid supplementation did not correct these developmental deficits. These results highlight the importance of maternal folate status during lactation in the delivery of adequate folate amounts to the infant.

The similar hepatic folate concentrations in both ND and FD pups on PND 10 indicate that maternal reserves were providing adequate folate amounts in the milk to all pups during the time these pups were feeding only on maternal milk. The dramatically lower hepatic folate concentrations on PND 23 in pups on the FD diet compared with those in pups on the ND reflect decreased folate supply from the mother as well as from the diet between PND 10 and 23. Brain folate concentrations, on the other hand, were $70 \%$ lower on PND 23 than on PND 10 in both the ND and FD groups and did not differ between the two groups. This may reflect high brain folate accumulation during fetal brain development followed by increased utilisation during early postnatal development that decreases it to adult brain levels by PND 23. The lack of difference in brain folate concentrations at this point between the two groups is consistent with previous observations in rats fed a FD diet and could be attributed to the conservation of brain folate by increased polyglutamation $^{(20,21)}$. Additionally, the unidirectional folate transport system via folate receptors that are highly expressed in the choroid plexus ${ }^{(22)}$, as opposed to the bidirectional reduced folate carrier transport system in other peripheral tissues ${ }^{(23)}$, is also likely to contribute to brain folate preservation.
In conditions of folate deficiency, tissues where folate receptors are highly expressed are also more resistant to folate loss. Despite apparently normal brain folate concentrations in the FD group, significantly elevated homocysteine concentrations in various regions indicated metabolic folate deficiency. The high brain homocysteine concentrations may have originated from other tissues and crossed the blood-brain barrier. Elevated homocysteine concentrations reduce the integrity of the bloodbrain barrier ${ }^{(24,25)}$ and methyl-deficient diets have previously been reported to be associated with homocysteine accumulation in the brain ${ }^{(26)}$. Switching the pups on the FD diet to a low-folate diet or a high-folate diet on PND 23 resulted in a tendency for increased hepatic and brain folate concentrations by PND 70 in the low-folate diet group and to significantly increased concentrations in the high-folate diet group.

Behavioural deficits were identified in pups reared on the FD diet regardless of being switched to a low-folate diet or a high-folate diet on PND 23. These ranged from anxietyand fear-related deficits to varying severity of memory and set-shifting impairments. Set shifting is defined as cognitive flexibility. It allows for goal-oriented behaviour to solve a problem or respond to a situation and is a component of executive functions. The frontal cortex and the hippocampus are involved in this function. Set shifting and higher-level cognitive abilities are impaired in numerous psychiatric and developmental disorders. Impaired set shifting indicates major deficits in conflict avoidance. It is a common characteristic of developmental disorders including autism and is likely 


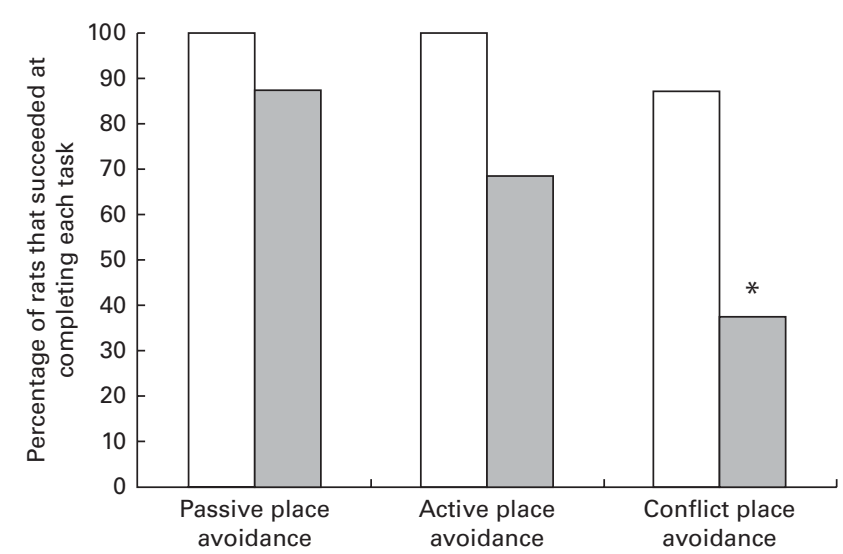

Fig. 6. Percentage of rats that successfully completed the place avoidance tasks. Rats failing the previous task were not tested for subsequent tasks, but were included in the calculation of the percentage of rats that failed the test. $\square$, The control group, rats on a normal diet throughout the weaning and post-weaning periods ( $n 8)$; $\square$, the experimental group, rats on a folatedeficient diet until postnatal day 23 and then switched to a low-folate diet or a high-folate diet $(n 16)$. * Percentage value was significantly different from that of the normal diet group $(P<0.05)$.

to involve multiple regions of the brain ${ }^{(27)}$. The observed set shifting deficits did not respond to folate supplementation after PND 23, indicating that they were likely to be permanent. A previous study has reported developmental deficits in the hippocampus in the offspring of mice that were folate deficient from gestational day 11 to gestational day 17 . Fewer neural progenitor cells and more apoptotic cells were observed on gestational day 17 in the hippocampus of the offspring. Choline supplementation partially corrected these defects $^{(28)}$. One of the consequences of abnormal methionine cycle activity due to folate deficiency might be epigenetic effects such as reduced DNA methylation ${ }^{(29)}$, leading to alterations in gene expression or function. Brain homocysteine accumulation in the pups reared on the FD diet may also have affected cognitive development. Homocysteine accumulation in neuronal cells has been reported to be associated with homocysteinylation of proteins contributing to altered differentiation, transport and plasticity ${ }^{(30)}$. Homocysteine accumulation was also observed in the hippocampus, which is involved in spatial working memory and consolidation of information (long-term memory). This brain region encodes and organises information from previous relationships on positions and marks (allothetic place information) to integrate and contribute to spatial and associative learning ${ }^{(17,31)}$ and involves the CA1 (Cornus Ammonis 1) and CA3 (Cornus Ammonis 3) fields of the hippocampus ${ }^{(32)}$.

The experimental group of rats in the present study exhibited an increase in the number of self-groomings and spent more time in the peripheral zone compared with the control group in the open field test. This behaviour was not corrected by folate supplementation after weaning. Increased episodes and duration of self-grooming in the open field test are characteristic of an experimental model of anxiety ${ }^{(33)}$ and autism ${ }^{(34)}$. The exact pathology underlying this behavioural presentation is not known; it is characteristic of stereotypy observed in many neurological disorders with potential involvement of the hypothalamus, limbic system and basal ganglia ${ }^{(35)}$. However, high-folic acid supplementation after weaning may have some corrective role as rats on the high-folate diet spent less time in peripheral area compared with those on the low-folate diet.

The present study showed that brain folate concentrations were not affected by folate deficiency during the weaning period in rat pups. However, transient folate deficiency during this period had long-term consequences on behaviour and learning in adult life that were not corrected by postweaning folic acid supplementation. This provides evidence in support of a critical role for folate in functional refinement of the brain during the postnatal period. In recent years, there has been increased interest in potential effects of maternal folate status during pregnancy on fetal brain development. In humans, low maternal folate status during the first trimester of pregnancy is known to cause behavioural deficits in their children $^{(36-38)}$. The neurological and/or behavioural evaluation of children in these studies was done at different ages, ranging from 11 months to 10 years. Various processes such

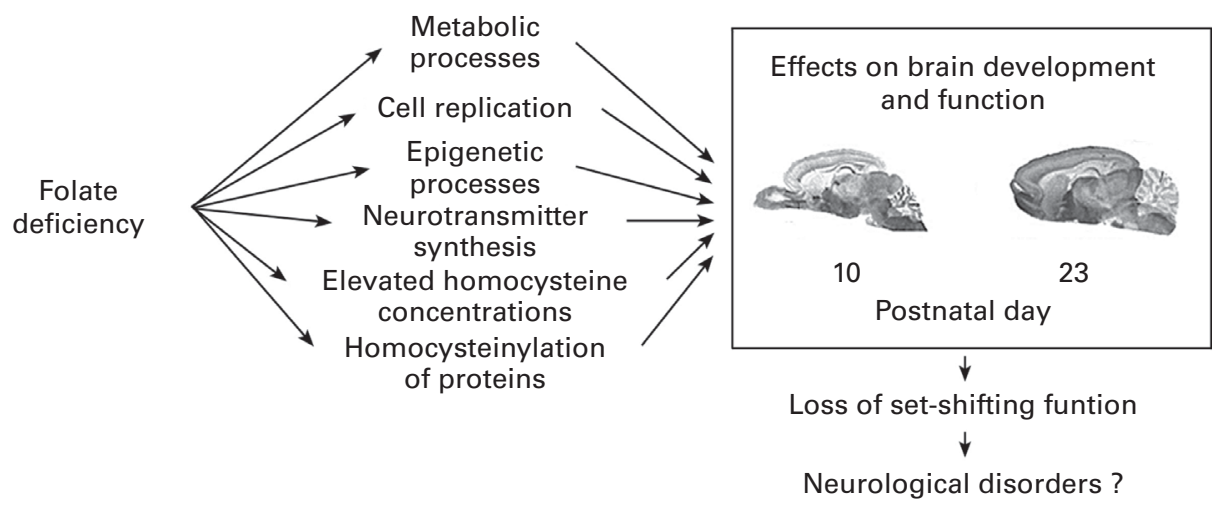

Autism spectrum disorders?

Fig. 7. Effects of folate on brain development and function. During postnatal development, the brain undergoes major structural refinement and grows during the $23 \mathrm{~d}$ of the weaning period. Folate is likely to influence and guide these events. The learning and memory deficits observed in rats rendered folate deficient during a narrow window between postnatal days 10 and 23 indicate that folate plays a major role in the structural and functional refinement of the brain of infants even during this late period. 
as myelination in humans begin in the third trimester of pregnancy, while in the rat this occurs during the weaning period $^{(5)}$. It is well recognised that folate deficiency during early pregnancy could lead to maldevelopment of the fetal brain, and a recent study has suggested that inadequate folate supply during pregnancy may increase the risk of autism and related neurodevelopmental disorders in the offspring ${ }^{(39)}$. The rat brain almost doubles in size between PND 10 and $24^{(40,41)}$, and this is also the period during which remodelling of the neuronal population and establishment of new connections occur in the cerebral cortex and hippocampus ${ }^{(42)}$. Deficiency of a critical nutrient such as folate is likely to affect a cascade of metabolic processes including DNA synthesis and cell replication, methylations, gene expression and neurotransmitter synthesis (Fig. 7). A previous study has suggested that serotonergic and dopaminergic systems in mice are affected by a $38 \mathrm{~d}$ FD dietary intervention ${ }^{(43)}$. These changes could lead to structural alterations that ultimately affect brain function.

In humans, folic acid supplementation has proven to be beneficial for preventing numerous pregnancy-related complications and fetal malformations ${ }^{(44,45)}$. In light of the observations made in the present study, adequate folate supplementation during the postnatal period may be necessary to ensure adequate folate delivery to the infant during the breast-feeding period. However, current prenatal folic acid supplementation levels may not be adequate for women positive for the folate receptor autoantibody ${ }^{(46,47)}$, and therefore identifying this population and treating with pharmacological doses of folinic acid may be necessary. Although this strategy has not been tested in pregnant women positive for the folate receptor autoantibody, it has proven to be beneficial in children with cerebral folate deficiency and autism ${ }^{(48-50)}$. Whether inadequate folate delivery to the fetus during pregnancy or to the infant during the first few years of life contributes to the spectrum of developmental disorders will need to be determined by additional studies in the form of large-scale clinical trials. Human studies should investigate the effect of maternal folate supplementation during pregnancy and lactation on developmental outcomes in children.

\section{Acknowledgements}

The present study received support from the Comissionat per a Universitats i Recerca del Departament d'Innovació, Universitats i Empresa de la Generalitat de Catalunya, through a postdoctoral fellowship to M. I. B.-Z. and from Autism Speaks through funding to E. V. Q.

The authors' contributions are as follows: E. V. Q. and J. M. S. designed the study; M. I. B.-Z. and J. M. S. conducted the study; S. G. A. B. and P. J. B. provided guidance in behavioural studies; M. I. B.-Z., J. M. S., M. M. M. and J. D. F.-B. analysed the data; M. I. B.-Z. and E. V. Q. wrote the article with input from J. M. S., P. J. B. and M. M. M.; E. V. Q. had primary responsibility for the final content. All authors read and approved the final manuscript.

None of the authors has any conflicts of interest to declare.

\section{References}

1. Wagner C (1995) Biochemical role of folate in cellular metabolism. In Folate in Health and Disease, 1st ed. [LB Bailey, editor]. New York: Marcel Dekker, Inc.

2. Scholl TO \& Johnson WG (2000) Folic acid: influence on the outcome of pregnancy. Am J Clin Nutr 71, 1295S-1303S.

3. Tamura $\mathrm{T}$ \& Picciano MF (2006) Folate and human reproduction. Am J Clin Nutr 83, 993-1016.

4. Taparia S, Gelineau-van Waes J, Rosenquist TH, et al. (2007) Importance of folate-homocysteine homeostasis during early embryonic development. Clin Chem Lab Med 45 , $1717-1727$.

5. Morgane PJ, Mokler DJ \& Galler JR (2002) Effects of prenatal protein malnutrition on the hippocampal formation. Neurosci Biobehav Rev 26, 471-483.

6. Stead JD, Neal C, Meng F, et al. (2006) Transcriptional profiling of the developing rat brain reveals that the most dramatic regional differentiation in gene expression occurs postpartum. J Neurosci 26, 345-353.

7. Craciunescu CN, Brown EC, Mar MH, et al. (2004) Folic acid deficiency during late gestation decreases progenitor cell proliferation and increases apoptosis in fetal mouse brain. J Nutr 134, 162-166.

8. Guéant JL, Namour F, Guéant-Rodriguez RM, et al. (2013) Folate and fetal programming: a play in epigenomics? Trends Endocrinol Metab 24, 279-289.

9. Breimer LH \& Nilsson TK (2012) Has folate a role in the developing nervous system after birth and not just during embryogenesis and gestation? Scand J Clin Lab Invest 72, 185-191.

10. Daval JL, Blaise S \& Guéant JL (2009) Vitamin B deficiency causes neural cell loss and cognitive impairment in the developing rat. Proc Natl Acad Sci US A 106, E1; author reply E2.

11. Ad hoc Committee of the American Institute of Nutrition (1977) Report of the American Institute of Nutrition ad hoc Committee on Standards for Nutritional Studies. J Nutr $\mathbf{1 0 7}$ 1340-1348.

12. Rothenberg SP, DaCosta M \& Rosenberg Z (1972) A radioassay for serum folate: use of a two-phase sequentialincubation, ligand-binding system. $N$ Engl J Med 286, $1335-1339$.

13. Swanson LW (1995) Mapping the human brain: past, present, and future. Trends Neurosci 18, 471-474.

14. Kalisch R, Schubert M, Jacob W, et al. (2006) Anxiety and hippocampus volume in the rat. Neuropsychopharmacology 31, 925-932.

15. Walsh RN \& Cummins RA (1976) The Open-Field Test: a critical review. Psychol Bull 83, 482-504.

16. Cimadevilla JM, Kaminsky Y, Fenton A, et al. (2000) Passive and active place avoidance as a tool of spatial memory research in rats. $J$ Neurosci Methods 102, 155-164.

17. Wesierska M, Dockery C \& Fenton AA (2005) Beyond memory, navigation, and inhibition: behavioural evidence for hippocampus-dependent cognitive coordination in the rat. J Neurosci 25, 2413-2419.

18. Cimadevilla JM, Fenton AA \& Bures J (2001) New spatial cognition tests for mice: passive place avoidance on stable and active place avoidance on rotating arenas. Brain Res Bull 54, 559-563.

19. Wesierska M, Adamska I \& Malinowska M (2009) Retrosplenial cortex lesion affected segregation of spatial information in place avoidance task in the rat. Neurobiol Learn Mem 91, 41-49. 
20. Ward GJ \& Nixon PF (1990) Modulation of pteroylpolyglutamate concentration and length in response to altered folate nutrition in a comprehensive range of rat tissues. $J$ Nutr 120, 476-484.

21. Eisenga BH, Collins TD \& McMartin KE (1992) Incorporation of ${ }^{3} \mathrm{H}$-label from folic acid is tissue-dependent in folatedeficient rats. J Nutr 122, 977-985.

22. Holm J, Hansen SI, Høier-Madsen M, et al. (1991) Highaffinity folate binding in human choroid plexus. Characterization of radioligand binding, immunoreactivity, molecular heterogeneity and hydrophobic domain of the binding protein. Biochem J 15, 267-271.

23. Sirotnak FM \& Tolner B (1999) Carrier-mediated membrane transport of folates in mammalian cells. Annu Rev Nutr $\mathbf{1 9}$ 91-122.

24. Kamath AF, Chauhan AK, Kisucka J, et al. (2006) Elevated levels of homocysteine compromise blood-brain barrier integrity in mice. Blood 107, 591-593.

25. Beard RS, Reynolds JJ \& Bearden SE (2011) Hyperhomocysteinemia increases permeability of the blood-brain barrier by NMDA receptor-dependent regulation of adherens and tight junctions. Blood 118, 2007-2014.

26. Blaise SA, Nédélec E, Schroeder H, et al. (2007) Gestational vitamin B deficiency leads to homocysteine-associated brain apoptosis and alters neurobehavioural development in rats. Am J Pathol 170, 667-679.

27. Periáñez JA, Maestú F, Barceló F, et al. (2004) Spatiotemporal brain dynamics during preparatory set shifting: MEG evidence. Neuroimage 21, 687-695.

28. Craciunescu CN, Johnson AR \& Zeisel SH (2010) Dietary choline reverses some, but not all, effects of folate deficiency on neurogenesis and apoptosis in fetal mouse brain. J Nutr 140, 1162-1166.

29. Duthie SJ, Narayanan S, Brand GM, et al. (2002) Impact of folate deficiency on DNA stability. J Nutr 132, 2444-2449.

30. Akchiche N, Bossenmeyer-Pourié C, Kerek R, et al. (2012) Homocysteinylation of neuronal proteins contributes to folate deficiency-associated alterations of differentiation, vesicular transport, and plasticity in hippocampal neuronal cells. FASEB J 26, 3980-3992.

31. Cimadevilla JM, Wesierska M, Fenton AA, et al. (2001) Inactivating one hippocampus impairs avoidance of a stable room-defined place during dissociation of arena cues from room cues by rotation of the arena. Proc Natl Acad Sci U S A 98, 3531-3536.

32. Stubley-Weatherly L, Harding JW \& Wright JW (1996) Effects of discrete kainic acid-induced hippocampal lesions on spatial and contextual learning and memory in rats. Brain Res 716, 29-38.

33. Kalueff AV \& Tuohimaa P (2004) Experimental modeling of anxiety and depression. Acta Neurobiol Exp (Wars) 64, 439-448.

34. Silverman JL, Tolu SS, Barkan CL, et al. (2010) Repetitive self-grooming behaviour in the BTBR mouse model of autism is blocked by the mGluR5 antagonist MPEP. Neuropsychopharmacology 35, 976-989.
35. Kalueff AV, Lou YR, Laaksi I, et al. (2004) Increased grooming behaviour in mice lacking vitamin D receptors. Physiol Behav 82, 405-409.

36. Holmes-Siedle M, Dennis J, Lindenbaum RH, et al. (1992) Long term effects of periconceptional multivitamin supplements for prevention of neural tube defects: a seven to 10 year follow up. Arch Dis Child 67, 1436-1441.

37. Dobó M \& Czeizel AE (1998) Long-term somatic and mental development of children after periconceptional multivitamin supplementation. Eur J Pediatr 157, 719-723.

38. Schlotz W, Jones A, Phillips DI, et al. (2010) Lower maternal folate status in early pregnancy is associated with childhood hyperactivity and peer problems in offspring. J Child Psychol Psychiatry 51, 594-602.

39. Surén P, Roth C, Bresnahan M, et al. (2013) Association between maternal use of folic acid supplements and risk of autism spectrum disorders in children. JAMA 309, 570-577.

40. Calabrese E, Johnson GA \& Watson C (2013) An ontologybased segmentation scheme for tracking postnatal changes in the developing rodent brain with MRI. Neuroimage $\mathbf{6 7}$, 375-384.

41. Calabrese E, Badea A, Watson C, et al. (2013) A quantitative magnetic resonance histology atlas of postnatal rat brain development with regional estimates of growth and variability. Neuroimage 71, 196-206.

42. Bandeira F, Lent R \& Herculano-Houzel S (2009) Changing numbers of neuronal and non-neuronal cells underlie postnatal brain growth in the rat. Proc Natl Acad Sci US A 106, 14108-14113.

43. Gospe SM, Gietzen DW, Summers PJ, et al. (1995) Behavioural and neurochemical changes in folate-deficient mice. Physiol Behav 58, 935-941.

44. Wald NJ (1994) Folic acid and neural tube defects: the current evidence and implications for prevention. Ciba Found Symp 181, 192-208, discussion 208-211.

45. Daly LE, Kirke PN, Molloy A, et al. (1995) Folate levels and neural tube defects. Implications for prevention. JAMA 274, 1698-1702.

46. Rothenberg SP, da Costa MP, Sequeira JM, et al. (2004) Autoantibodies against folate receptors in women with a pregnancy complicated by a neural-tube defect. $N$ Engl J Med 350, 134-142.

47. Cabrera RM, Shaw GM, Ballard JL, et al. (2008) Autoantibodies to folate receptor during pregnancy and neural tube defect risk. J Reprod Immunol 79, 85-92.

48. Ramaekers VT, Sequeira JM, Blau N, et al. (2008) A milk-free diet downregulates folate receptor autoimmunity in cerebral folate deficiency syndrome. Dev Med Child Neurol 50, 346-352.

49. Ramaekers VT, Quadros EV \& Sequeira JM (2013) Role of folate receptor autoantibodies in infantile autism. Mol Psychiatry 18, 270-271.

50. Frye RE, Sequeira JM, Quadros EV, et al. (2013) Cerebral folate receptor autoantibodies in autism spectrum disorder. Mol Psychiatry 18, 369-381. 